LIAMES, Campinas, SP, v. 20, 1-2, e020018, 2020

\title{
Acknowledgment to Reviewers - LIAMES vol. 20, 2020
}

\author{
Angel Corbera Mori \\ Universidade Estadual de Campinas, Brasil \\ https://orcid.org/0000-0003-1712-6550 \\ Editor
}

We present below the alphabetical list of the names and institutions of blind peer reviewers who have dedicated their time and expertise to the Journal American Indigenous Languages (LIAMES), volume 20, evaluating articles submitted during the year of 2020. The Editorial Committee thanks to all of them for their commitment in making the LIAMES a Journal of high academic quality.

Adam Roth Singerman (University of Chicago, Estados Unidos da América)

Aldir Santos de Paula (Universidade Federal de Alagoas, Brasil)

Ana Carla dos Santos Bruno (Instituto Nacional de Pesquisas da Amazônia, Brasil)

Ana Quadros Gomes (Universidade Federal do Rio de Janeiro, Brasil)

Andrés Pablo Salanova (University of Ottawa, Canada)

Andrés Romero Figueroa (Universidad Católica Andrés Bello, Venezuela)

Beatriz Gualdieri (Universidad Nacional de Luján, Argentina)

Bernart Bardagil (University of California, Berkeley, Estados Unidos da América)

Carolina González (Florida State University, Estados Unidos da América)

Cláudio André Cavalcanti Couto (universidade Estadual de Campinas/CAPES, Brasil)

Fernando Zúñiga (University of Bern, Suíça)

Francisco Queixalós (Centre National de Recherche Scientifique, França)

Glauber Romling da Silva (Universidade Federal do Amapá, Brasil)

Gustavo de Godoy e Silva (Fundação Nacional do Índio/FunAI, Brasil)

Gustavo Solís (Universidad Nacional Mayor de San Marcos, Perú)

Hebe Alicia González (Universidad Nacional de San Juan/ CONICET, Argentina)

Hein van der Voort (Museu Paraense Emílio Goeldi, Brasil)

Karolin Obert (The University of Texas at Austin, Estados Unidos da América)

Kayron Campos Beviláqua (Instituto Federal de Santa Catarina, Brasil)

Luciana Sánchez-Mandes (Universidade Federal Fluminense, Brasil)

Luis Miguel Rojas-Berscia (University of Queensland, Brisbane, Australia)

DOI: http://dx.doi.org/10.20396/liames.v20i0.8663679 
Magnun Rochel Madruga (Universidade Federal de Minas Gerais, Brasil)

Maia Filomena Spatti Sandalo (Universidade Estadual de Campinas, Brasil)

María Emilia Montes Rodríguez (Universidad Nacional de Colombia, Colombia)

Mário André Coelho da Silva (Pós.Doc - Universidade Estadual de Campinas, Brasil)

Mateus Cruz Maciel de Carvalho (Instituto Federal de Educação, Ciência e Tecnologia de São Paulo, Brasil)

Maxwell Gomes Miranda (Universidade Federal de Mato Grosso, Brasil)

Pafnuncio Antonio Ramos (Universidad de Sonora/COnACYT, México)

Pilar Chamorro Fernández (University of Georgia, Estados Unidos da América)

Renato Miguel Basso (Universidade Federal de São Carlos, Brasil)

Ricardo Campos Castro (Pós Doc. FaPesp/UniCAmP / University of Georgia, Estados Unidos da América)

Roberta Pires de Oliveira (Universidade Federal de San Catarina, Brasil)

Suzi Lima (University of Toronto, Canada)

Thiago Costa Chacon (Universidade de Brasília, Brasil)

Thomas D. Finbow (Universidade de São Paulo, Brasil)

Wilmar Rocha D’Angelis (Universidade Estadual de Campinas, Brasil)

Wolf Dietrich (Universidade de Münster, Alemanha)

Zachary O'Hagan (University of California, Berkeley, Estados Unidos da América) 